\title{
CULTURA
}

MATERIAL

E PESQUISA

MUSEOLÓGICA:

\author{
UM ESTUDO RELACIONADO AO PATRIMÔNIO \\ CULTURAL DE CIÊNCIA E TECNOLOGIA NO MUSEU \\ DA GEODIVERSIDADE (IGEO-UFRJ)
}

GEÓRGIA RAISA RAMOS ALBUQUERQUE, UNIVERSIDADE FEDERAL DO RIO DE JANEIRO, RIO DE JANEIRO, RIO DE JANEIRO, BRASIL

Graduanda em História pela Universidade Federal do Rio de Janeiro (UFRJ). Bolsista de Iniciação Científica (PIBIC-UFRJ) do Museu da Geodiversidade (IGEO-UFRJ).

E-mail: georgia.r.albuquerque@gmail.com

LUIZ FELIPE LIMA FERREIRA, UNIVERSIDADE FEDERAL DO RIO DE JANEIRO, RIO DE JANEIRO, RIO DE JANEIRO, BRASIL

Museólogo e mestre pelo Programa de Pós-Graduação em Divulgação da Ciência, Tecnologia e Saúde (COC-FIOCRUZ). É pesquisador colaborador do Museu da Geodiversidade.

E-mail: luizfelipe.museusemuseologia@gmail.com

ALINE ROCHA DE SOUZA FERREIRA DE CASTRO, UNIVERSIDADE FEDERAL DO RIO DE JANEIRO, RIO DE JANEIRO, RIO DE JANEIRO, BRASIL

Museóloga, Mestre em Museologia e Patrimônio (UNIRIO-MAST) e Doutora em Geologia pela Universidade Federal do Rio de Janeiro. E-mail: alinecastro@igeo.ufrj.br. E-mail: alinecastro@igeo.ufrj.br

RECEBIDO

DOI

10/07/2020

APROVADO

http://dx.doi.org/10.11606/issn.1980-4466.v15i30p192-208

$20 / 12 / 2020$ 


\section{CULTURA MATERIAL E PESQUISA MUSEOLÓGICA: UM ESTUDO RELACIONADO AO PATRIMÔNIO CULTURAL DE CIÊNCIA E TECNOLOGIA NO MUSEU DA GEODIVERSIDADE (IGEO-UFRJ)}

GEÓRGIA RAISA RAMOS ALBUQUERQUE, LUIZ FELIPE LIMA FERREIRA, ALINE ROCHA DE SOUZA FERREIRA DE CASTRO

\section{RESUMO}

Este trabalho apresenta, a partir da pesquisa museológica, a história da construção do conhecimento científico produzido pela Universidade Federal do Rio de Janeiro (UFRJ), através de objetos encontrados nos laboratórios do Instituto de Geociências (IGeo). Para tanto, o Museu da Geodiversidade (IGeo-UFRJ) por meio do projeto "Objetos de ciência e tecnologia do Instituto de Geociências (IGeo) da Universidade Federal do Rio de Janeiro: pesquisa e identificação de um patrimônio a ser descoberto", propôs uma pesquisa para levantar, catalogar e preservar objetos e instrumentos científicos e didáticos que ajudaram na produção e divulgação do conhecimento acadêmico. A pesquisa levantou, até o momento, informações sobre 164 itens, dentre os quais dois objetos distintos destacaram-se durante as atividades: um barógrafo centenário, modelo Anciennes Maison Richard Frères Jules Richard, doação do professor José Marques do Departamento de Meteorologia UFRJ, o objeto mais antigo da coleção; e uma balança eletrônica de sedimentação Sartorius, adquirida pelo Instituto de Geociências em 1970, sendo o elemento mais bem documentado até então. Apesar da relevância que a pesquisa revelou, esse patrimônio necessita de maior atenção por parte da instituição, que deve assegurar um local de guarda adequado, além de incentivos para que a pesquisa continue, pois muitos outros objetos podem se destacar no conjunto já levantado. Verificamos que o projeto de pesquisa foi fundamental para essas descobertas que possibilitarão a preservação da memória institucional e a musealização dessa parte do patrimônio cultural de ciência e tecnologia da UFRJ.

\section{PALAVRAS-CHAVE}

Pesquisa museológica, Documentação museológica, Patrimônio cultural de ciência e tecnologia, Museus universitários. 


\section{MATERIAL CULTURE AND MUSEUM RESEARCH: A STUDY RELATED TO SCIENCE AND TECHNOLOGY CULTURAL HERITAGE IN GEODIVERSITY MUSEUM (IGEO-UFRJ)}

GEÓRGIA RAISA RAMOS ALBUQUERQUE, LUIZ FELIPE LIMA FERREIRA, ALINE ROCHA DE SOUZA FERREIRA DE CASTRO

\section{ABSTRACT}

This study was based on museological research, and presents the history of the scientific knowledge produced by the Federal University of Rio de Janeiro - UFRJ (Brazil) from some objects found in the Geosciences Institute's laboratories. For this, the Geodiversity Museum, through the project "Science and Technology Objects of the Geosciences Institute (IGeo) of the Federal University of Rio de Janeiro: Research and Identification of a Heritage to be Discovered", proposes a research to raise, catalogue and preserve scientific and educational objects and instruments that helped in the production and dissemination of academic knowledge. Despite having approximately one hundred and sixty-four items catalogued, two distinct objects stood out during the activities: one centenary barometer, brand Anciennes Maison Richard Frères Jules Richard, the collection's oldest object, a donation from professor José Marques of the UFRJ Meteorology Department ; and one electronic sedimentation scale by Sartorius, acquired by the Geosciences Institute in 1970, being the most well documented item so far. Despite the relevance that this research has revealed, this collection needs more attention from the institution in order to ensure an adequate place to safeguard it, in addition to a greater support so it may continue, as many other objects can stand out in the preexisting set. Thus, we have verified that this research project was essencial for these discoveries to occur and that it will enable the institutional memory's preservation and musealization of this part of UFRJ's cultural heritage of science and technology.

\section{KEYWORDS}

Museological research, Museological documentation, Science and technology cultural heritage, University museums. 


\section{INTRODUÇÃO}

Criado em 2007, o Museu da Geodiversidade está vinculado ao Instituto de Geociências da Universidade Federal do Rio de Janeiro (IGeo-UFRJ). Sendo um museu universitário, desempenha importante papel na preservação da história geológica e na divulgação científica e é responsável pela guarda e organização de um acervo de fósseis, rochas, minerais, meteoritos e reconstituições que compõem sua exposição de longa duração (CASTRO; GRANATO, 2018, p. 5710). Além das coleções de paleontologia, mineralogia e geologia, existem outros itens que podem se configurar como acervo museológico, mas que ainda não estão contemplados como patrimônio na unidade em que o museu está localizado. É o caso dos objetos relacionados à ciência e tecnologia (C\&T) que estão dispersos por diversos laboratórios, conforme comprovam as etiquetas e marcas que deles fazem parte. Esses objetos muitas vezes são descartados sem receberem o tratamento e o estudo aprofundados que poderiam resultar na sua ressignificação como patrimônio.

De acordo com Taub (2009), ainda não há uma origem definitiva para o conceito de objeto científico. Embora existam menções que datam do final do século XVII, na França, e no início do século XIX, na Alemanha, o termo "científico" só passou a ser utilizado para objetos do século XIX e início do século XX, quando a ciência ganhou a forma como a compreendemos atualmente. Apesar dos debates, compreende-se que, ao considerar 
o contexto de uso prático, tais objetos estavam relacionados diretamente às atividades científicas, principalmente às experiências. Entretanto, a autora adverte que delimitar o que são atividades científicas não é algo simples e, portanto, essas associações seguem o mesmo desafio.

Assim, temos o conceito de patrimônio cultural de ciência e tecnologia, que é bastante amplo e vai além dos instrumentos, incluindo elementos que ajudaram a construir o conhecimento científico, como explicam Granato e Lourenço:

[...] o patrimônio cultural da C\&T inclui o conhecimento científico e tecnológico produzido pelo homem, além de todos aqueles objetos (inclusive documentos em suporte papel), utilizados em laboratórios, as coleções arqueológicas, etnográficas e espécimes das coleções biológicas e da Terra, que são testemunhos dos processos científicos e do desenvolvimento tecnológico. Também se incluem nesse grande conjunto as construções arquitetônicas produzidas com a funcionalidade de atender às necessidades desses processos e desenvolvimentos, por exemplo laboratórios, grandes equipamentos, observatórios etc. (GRANATO; LOURENÇO, 2011, p. 90).

Castro e Granato (2018) explicam que o reconhecimento do patrimônio cultural de ciência e tecnologia como tal é resultado da cooperação interdisciplinar das comunidades científicas interessadas na sua consolidação, preservação e divulgação, mas salientam que ainda há um longo percurso a ser trilhado para que isso ocorra, principalmente nas universidades.

O Museu de Astronomia e Ciências Afins (MAST) promoveu um levantamento nacional para apurar onde está esse patrimônio. Esse levantamento, além de apontar que o conhecimento especializado sobre o tema ainda é limitado, demonstrou que esses bens são frequentemente encontrados em universidades, sejam nos laboratórios ou nos museus universitários (GRANATO; SANTOS; MIRANDA, 2005). Nesse sentido, a formação de uma coleção que visa a preservar a história da ciência produzida pela Universidade Federal do Rio de Janeiro, com enfoque nas geociências, é fundamental para a preservação da memória desta universidade.

O Museu da Geodiversidade, por meio do projeto "Objetos de ciência e tecnologia do Instituto de Geociências (IGeo) da Universidade Federal do Rio de Janeiro: pesquisa e identificação de um patrimônio a ser descoberto" propôs levantar, catalogar e preservar objetos e instrumentos científicos e didáticos que 
ajudaram na produção e divulgação do conhecimento acadêmico. Nesse artigo serão apresentados alguns resultados alcançados por essa pesquisa.

Através da análise dos objetos, verificou-se que alguns possuem marcas e inscrições que remetem às origens dos cursos que compõem o Instituto de Geociências - Geologia, Geografia e Meteorologia - desde 1967. Tais legendas indicam a origem do objeto, o que possibilita situá-lo na ordem cronológica e analisar o investimento aplicado nas pesquisas dos laboratórios.

O curso de Geografia foi criado em 1935 e pertenceu à antiga Faculdade Nacional de Filosofia (FNFI), fundada em 1939 pelo então presidente Getúlio Vargas. Após sua extinção, em 1968, fragmentou-se em unidades ou departamentos da UFRJ, como foi o caso do Departamento de Geografia do IGeo. Já o Departamento de Geologia teve origem no curso de Geologia do Rio de Janeiro (1958), quinta unidade brasileira organizada pela Companhia de Formação de Geólogos (CAGE), que originou posteriormente a Escola Nacional de Geologia e, mais tarde, foi integrada à Universidade do Brasil, hoje Universidade Federal do Rio de Janeiro. De acordo com as informações presentes no site do Departamento de Meteorologia, o curso teve início na década de 1960, sendo o primeiro curso de formação superior na área e responsável pela formação dos primeiros meteorologistas do Brasil (CASTRO; GRANATO, 2018, p. 5710; UNIVERSIDADE FEDERAL DO RIO DE JANEIRO, s.d., s/p).

Assim sendo, a história do instituto, diretamente ligada à antiga Faculdade Nacional de Filosofia e conectada a outras faculdades e à Universidade do Brasil, ajuda a compreender como ocorreu a instalação de cursos universitários e a demanda pelo ensino de curso superior e pela pesquisa no país. Parte desta história pode ser contada por meio da análise dos objetos que contribuíram para a pesquisa e o ensino universitário ao longo tempo. São vestígios compostos por itens utilizados pelos pesquisadores e professores da instituição que precisam ser conhecidos e organizados em uma coleção que representará o elo entre o fazer da ciência no passado com o presente e o futuro. 


\section{METODOLOGIA}

A pesquisa museológica foi a abordagem escolhida para tratar os objetos de ciência e tecnologia localizados no Instituto de Geociências e que passarão a integrar o acervo do Museu da Geodiversidade, conforme os avanços da pesquisa. Segundo Desvallés e Mairesse (2013), a pesquisa museológica em coleções de museu consiste em investigar o conteúdo das coleções e se apoia nas áreas do conhecimento diretamente ligadas a esse conteúdo (história da arte, história, história da ciência etc.). Essa atividade tem por finalidade a documentação, preservação e comunicação das coleções.

Para atender aos objetivos do trabalho foi realizado um levantamento bibliográfico sobre o tema fundamentado na busca de periódicos e, ambiente eletrônico. A partir das fontes identificadas e das fontes secundárias foram realizados fichamentos do conteúdo para embasamento do texto.

Para a identificação dos objetos levantados utilizou-se como ferramenta principal o Thesaurus de acervos cientificos em língua portuguesa, documento que define a nomenclatura correta dos equipamentos e os categoriza em uma hierarquia sistemática de seu uso dentro das ciências. O Thesaurus foi elaborado entre 2006 e 2013 e é fruto de uma parceria entre instituições de Portugal e do Brasil, como o Museu Nacional de História Natural e da Ciência (Universidade de Lisboa) e o Museu de Astronomia e Ciências Afins do Rio de Janeiro (MAST). O projeto recebeu o apoio da Fundação para Ciência e Tecnologia (FCT) de Portugal e do Conselho Nacional de Desenvolvimento Científico e Tecnológico do Brasil (CNPq) (SILVA; DA SILVA; NUNES, 2016, p. 5). Apesar da riqueza metodológica do Thesaurus, esse recurso atualmente não contempla objetos didáticos, como os modelos em gesso ou madeira para uso em sala de aula para ilustrar, por exemplo, os processos geológicos. Para esses objetos contou-se com arquivos, sites de antiquários, manuais de instruções, catálogos de fabricantes e a experiência dos professores mais antigos.

Para manter o controle dos objetos reunidos até então, suas informações foram inseridas em uma ficha catalográfica desenvolvida no âmbito da pesquisa (CASTRO; GRANATO, 2018). A ficha funciona como um "documento de identidade" do objeto e é o material a ser consultado ao se manusear o item. Foi elaborada de acordo com as diretrizes da Cartilha de orientações gerais para preservação do patrimônio cultural de CઐT, desenvolvida pelo MAST (GRANATO et al., 2013). 
Para auxiliar no preenchimento das fichas também foram utilizados o Glossário de termos técnicos e o Manual de instruções para uso da futura coleção de ciência e tecnologia do Museu da Geodiversidade (IGEO/UFRJ), materiais desenvolvidos internamente e que funcionam também como tutorial para futuros pesquisadores da instituição.

\section{OS DESAFIOS PARA PRESERVAR OS OBJETOS DE C\&T}

Granato, Santos e Miranda (2005) enfatizaram a importância da preservação dos objetos de C\&T, que se tornam obsoletos cada vez mais cedo devido aos avanços tecnológicos, sendo descartados e substituídos por modelos mais atuais de forma acelerada. Nessa rotina da universidade, muitos objetos se perdem sem qualquer análise do ponto de vista museológico.

\footnotetext{
Os instrumentos científicos, em contrapartida, tornam-se obsoletos cada vez mais rápido e o desaparecimento do seu conteúdo documental pode constituir a perda de uma parte intrínseca do conhecimento. A noção de conservação aparece como uma primeira etapa necessária a garantir a salvaguarda desses instrumentos de caráter histórico (GRANATO; SANTOS; MIRANDA, 2005, p. 246).
}

No IGeo, esse procedimento não era diferente. Em seus laboratórios, dispersos pelos três departamentos, houve situações em que objetos foram substituídos por outros mais modernos e, sem nenhuma intervenção, foram descartados, conforme a rotina administrativa adotada pela universidade. Com a atuação do MGeo, um trabalho de conscientização vem sendo realizado buscando intervir no descarte e, por meio de pesquisa, identificar a relevância patrimonial desses objetos.

Outro desafio inerente à pesquisa de objetos científicos é descrito por Silva e Barboza (2012) no capítulo de introdução do livro Acervos de ciência e tecnologia no Brasil: preservação, história e divulgação, publicado pelo MAST. Segundo as autoras, as áreas que desenvolvem pesquisas e tecnologias de ponta possuem prioridade de recursos, resultando na escassez para os setores de conservação e restauro. Com isso, ocorre um processo que as autoras chamam de "canibalização" de objetos e dispositivos, no qual, ao reparar danos em determinados aparelhos, retiram-se peças de outros. Tal prática implica em duas problemáticas principais: a redução da vida útil do dispositivo e sua descaracterização, o que dificulta a identificação 
e o transforma em sucata.

[...] o "canibalismo" de equipamentos e dispositivos - isto é, a retirada de peças para aproveitamento em outros equipamentos com melhor perspectiva de utilização - é comum nessas instituições. Em consequência, não é só a vida útil desses objetos que é reduzida, com sua transformação em sucata, mas o próprio trabalho de identificação e intervenção por parte do conservador fica prejudicado (SILVA; BARBOZA, 2012, p. 15).

Por conta da diversidade e da abrangência do conhecimento científico, diversas áreas atuam no desenvolvimento de pesquisas como esta. Essa diversidade está representada nos acervos de ciência e tecnologia, nos quais se encontram objetos que fizeram parte de pesquisas relacionadas a diferentes linhas e disciplinas científicas. Em seu trabalho Conhecer para preservar: práticas de socialização de acervos em museus de ciência e tecnologia, Maria Augusta André (2012) expõe de modo objetivo a interdisciplinaridade da Museologia em relação às coleções de ciência e tecnologia:

A popularização da ciência, assim como a Museologia, têm caráter interdisciplinar, haja vista a aproximação com a História, a Biblioteconomia, a Filosofia e a Sociologia, só para citar as ciências humanas e sociais (ANDRÉ, 2012, p. 133).

O desafio de pesquisar a história por trás dos itens deve-se à interpretação de suas informações materiais e funcionais. Caso os objetos estejam muito deteriorados, com peças soltas e pouco material de referência, a sua identificação torna-se mais difícil (JULIÃO, 2006). Conforme Julião (2006) demonstra são muitos os desafios enfrentados e é necessário intervir rápido no processo para que os objetos se mantenham íntegros e não haja perda informacional sobre o seu uso. Essa perda informacional aconteceu com boa parte dos objetos de C\&T encontrados no IGeo e reunidos pelo Museu da Geodiversidade. Para suprimir essa perda, o trabalho de pesquisa tem sido essencial para verificar a importância desses objetos para a memória da instituição e, em consequência, a sua relevância como patrimônio. No entanto, ainda que viável, é importante destacar que todo esse processo é bastante complexo e muito demorado. 


\subsection{O caminho para conhecer os objetos de C\&T do Instituto de Geociências}

Para corroborar um dos papéis institucionais do Museu da Geodiversidade, vem sendo realizado o trabalho de levantamento, pesquisa, inventário e catalogação para o desenvolvimento de políticas de preservação dos objetos que podem vir a constituir a coleção de patrimônio cultural de ciência e tecnologia.

A identificação dos objetos e o preenchimento da ficha como um todo são realizados a partir da consulta ao Glossário de termos técnicos e manual de instruções para uso da futura coleção de ciência e tecnologia do Museu da Geodiversidade (IGEO/UFRJ), criado no âmbito da pesquisa para o museu. Tal material, assim como o Thesaurus de acervos científicos em Língua Portuguesa, tem como propósitos básicos uniformizar, informar e instruir futuros pesquisadores interessados no uso, produção e recuperação das informações dos objetos. Sendo assim, é um material fundamental para assegurar a continuidade da pesquisa. Portanto, também é o primeiro passo para iniciar uma política de documentação. Esse documento passa por atualizações e correções conforme a catalogação da coleção evolui, pois quanto mais objetos são catalogados mais questões demandam respostas, devido à sua diversidade. Cada objeto é registrado individualmente em uma ficha própria que serve para resguardar as informações dos itens. Assim, reduz-se o manuseio e contato ao máximo, respeitando as normas de conservação e garantindo a sua preservação.

Até o presente momento foram catalogados, total ou parcialmente, 164 objetos. Os objetos apurados compreendem instrumentos científicos de observação, medição e registro das áreas da física, química, meteorologia, mineralogia, geofísica e também instrumentos e materiais para ensino, tais como slides e projetores e modelos didáticos de ambientes, para dar dois exemplos.

De modo geral, o conjunto reunido possui mais objetos de um mesmo tipo que outros (cerca de quatro a seis itens), por exemplo, os barômetros, os teodolitos, os microscópios e as balanças, o que possibilita analisar a sua variedade de fábrica, a demanda para os laboratórios e também a evolução tecnológica. Nesse caso, as balanças da futura coleção mostram maior diversidade, podendo ser de um ou de dois pratos, com ou sem pesos inclusos, como elementos como o fiel e o cavaleiro entre outros. Em sua maioria, as balanças são de fabricação da marca Sartorius Werke. 


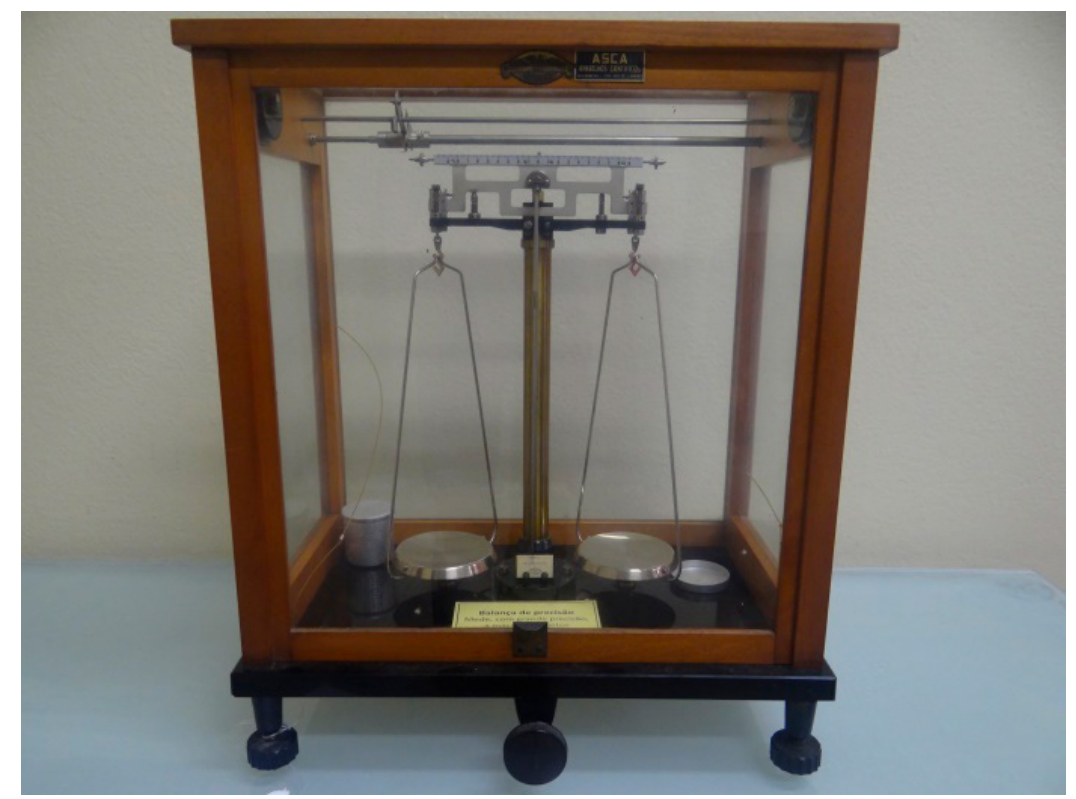

A priori, a identificação foi complexa, pois trabalhar com esse novo patrimônio requer saber que não há muitos materiais de referência disponíveis sobre esse tipo de bem cultural. Alguns objetos estão em fase de identificação, portanto, suas fichas são preenchidas com as informações que podemos obter a partir do próprio item, como materiais de fabricação, inscrições, legendas e estado de conservação. Como medida de conservação dos objetos e para facilitar a identificação e a pesquisa foi realizada a higienização mecânica dos objetos. A qualificação da equipe se deu por meio de treinamento realizado pela Coordenação de Museologia do MAST (Figuras 2 e 3)

Dentre os objetos registrados, dois foram selecionados - o barógrafo centenário, doação do professor José Marques, e uma balança eletrônica de sedimentação. A escolha desses itens seguiu alguns critérios para o início da pesquisa: o barógrafo é o instrumento mais antigo e estabelece o marco inicial da coleção no século XX e a balança é o elemento mais bem documentado até então, com todos os documentos de compra presentes no arquivo do Instituto de Geociências. 
FIGURA 2 (ESQ.)

Balança de um prato pré-higienização.

Fonte: Geórgia

Albuquerque (2018)

FIGURA 3 (DIR.)

Balança de um prato pós-higienização. Fotogragia: Geórgia Albuquerque (2018).
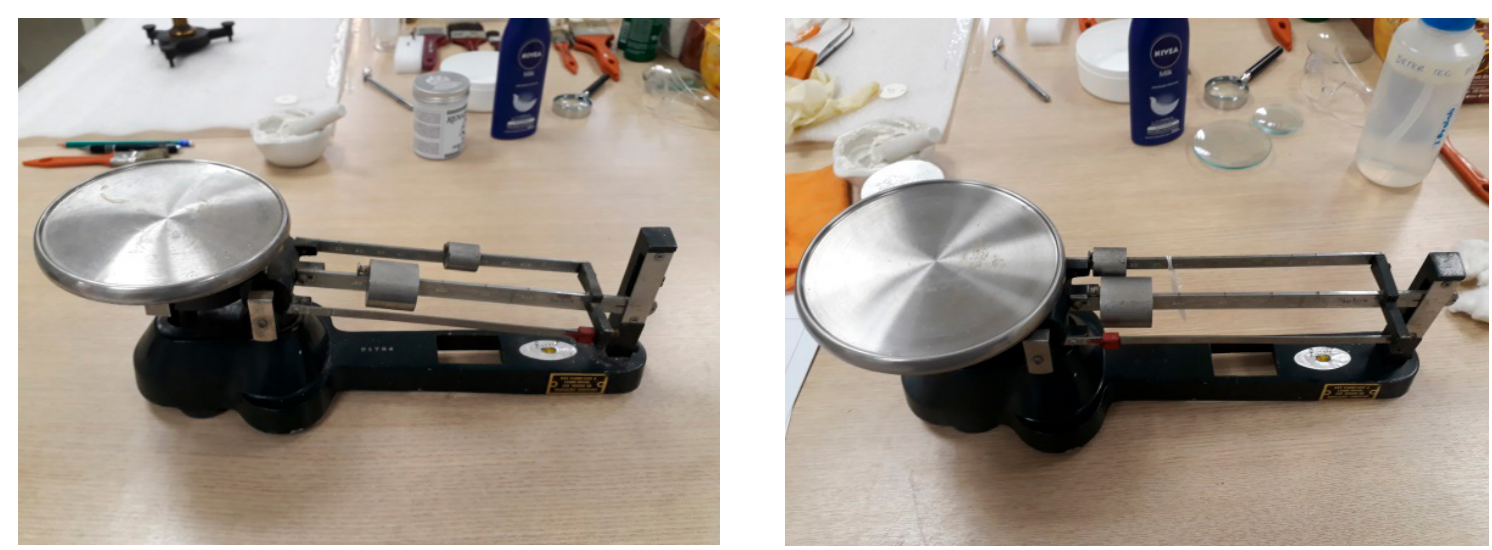

O barógrafo é utilizado em estações meteorológicas para medir e registrar continuamente as variações de pressão atmosférica. O equipamento presente na coleção do Museu da Geodiversidade é um modelo emblemático dentro da história científic: Anciennes Maison Richard Frères - Jules Richard (Figura 4), e sua produção data do início no século XX. Jules Richard trabalhou durante a década de 1870 na confecção de equipamentos para telégrafos. Oriundo de uma família fabricante de instrumentos científicos, em 1876, com a morte do pai, retornou ao negócio da família, e em 1882 consolidou parceria com seu irmão Max. Nascia assim a empresa Richard Frères, representada pelo logo RF, que, mesmo depois de dissolvida manteve a sigla original. Notória pela fabricação de barômetros aneroides e de gravação (barógrafo), logo expandiu sua produção para outros aparelhos de registro científico, tais como termômetros, anemômetros, pirômetros e diversas formas de medições elétricas, além de câmeras estereoscópicas e equipamentos de visualização. Após 30 anos de administração pessoal por Jules Richard, em 1921 a empresa tornou-se pública. Atualmente a companhia se chama JRI (SCIENCE MUSEUM COLLECTION, s.d.).

De acordo com as informações presentes nas etiquetas anexadas ao equipamento, ele pertenceu à Escola de Engenharia de Porto Alegre e, em algum momento ainda desconhecido, a sua guarda e posse foi transferida ao professor José Marques do Departamento de Meteorologia da UFRJ. Por outro lado, informações levantadas por meio da troca de mensagens eletrônicas com professores do instituto mostram que o barógrafo em questão pode ter pertencido à professora Ana Maria de Paiva Macedo Brandão, do 
Departamento de Geografia, hoje aposentada ${ }^{1}$. Houve tentativas de localizar a professora via contatos disponibilizados pelo Departamento Pessoal do IGeo e também pelo contato presente na Plataforma Lattes (CNPq), mas sem sucesso até a publicação desse artigo. Ainda de acordo com as informações disponibilizadas na Plataforma Lattes, as suas pesquisas concentravam-se na área de Geociências, atuando principalmente com clima urbano, educação ambiental, desenvolvimento sustentável e conforto térmico².

Durante a pesquisa no arquivo do Instituto de Geociências foram encontrados documentos referentes a uma balança eletrônica de sedimentação Sartorius Werke (Figura 5), adquirida pela unidade em 1970. O instrumento foi comprado em Hamburg, Alemanha, e foi transportado de navio em três embalagens: balança, estabilizador de voltagem e uma mesa antitrepidação, somando o valor de 9666,11 cruzeiros. Tais documentos formam um dossiê de 17 páginas, as quais descrevem detalhadamente todo o processo de origem, compra, transporte e destino do item. Portanto, mostra o panorama do investimento que se fez no instituto e a importância das pesquisas desenvolvidas ali.

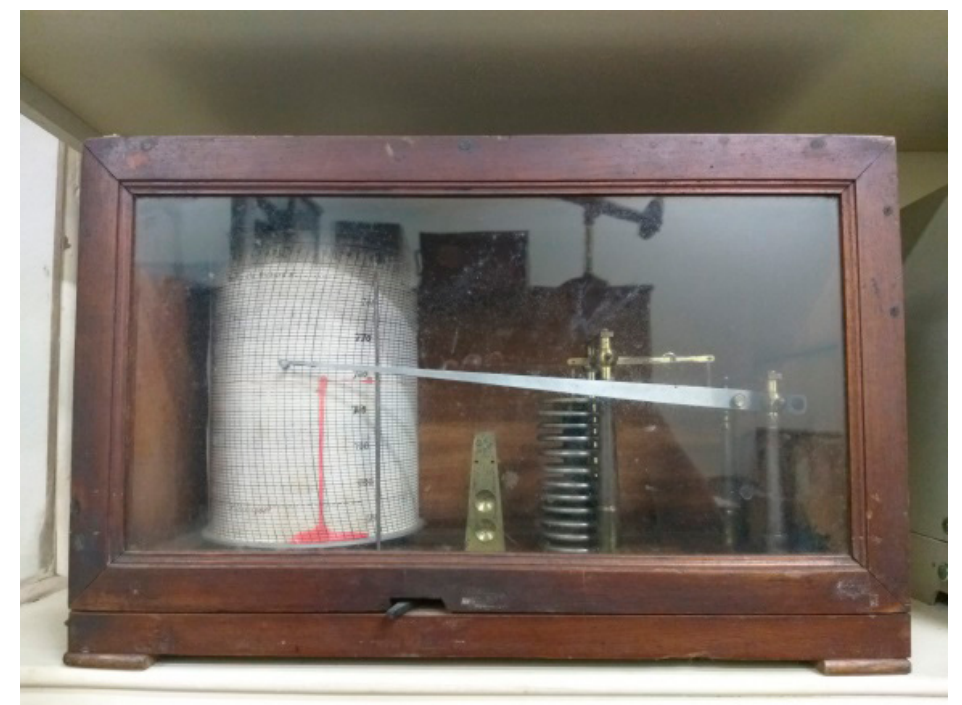

1 Conforme troca de mensagens eletrônicas realizadas no dia 14 de maio de 2020 com o prof. Luiz Maia, do Departamento de Meteorologia do Instituto de Geociências da UFRJ.

$2 \mathrm{O}$ currículo de Ana Maria de Paiva Macedo Brandão está disponível em: http://lattes.cnpq. br/1824293102476623. Acesso em: junho de 2020. 
FIGURA 5

Balança eletrônica de sedimentação.

Fonte: Geórgia

Albuquerque (2019).

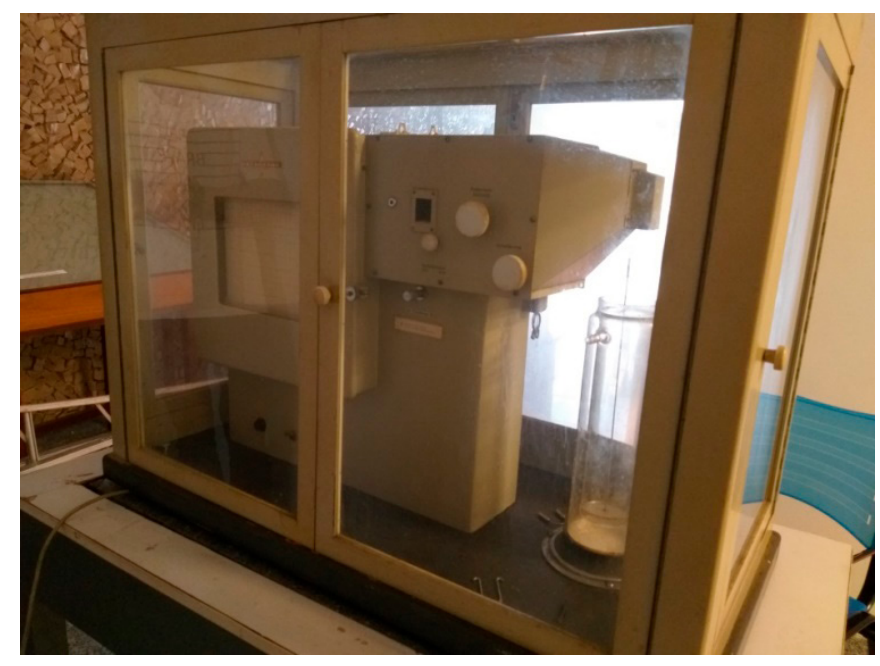

FIGURA 6

Nota fiscal referente à compra da balança de sedimentação.

Fonte: Arquivo

do Instituto de

Geociências.

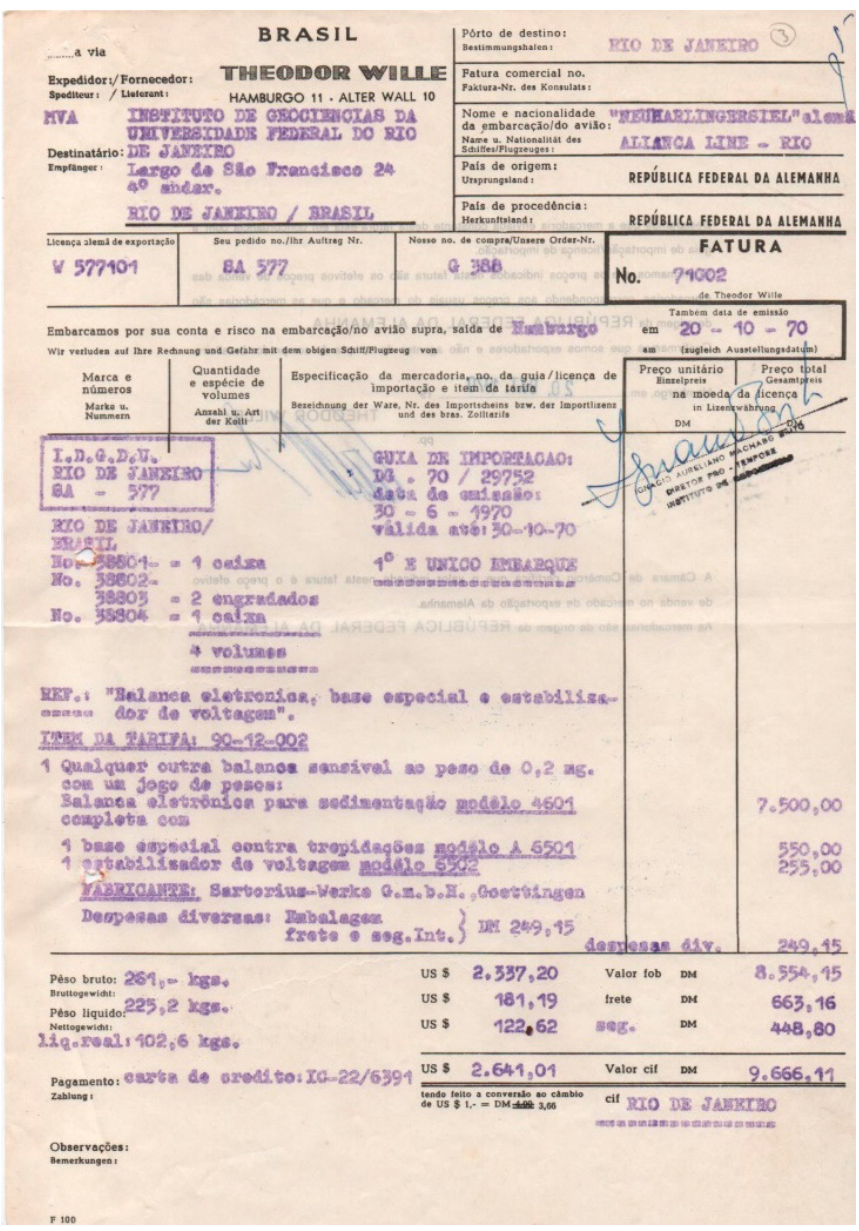


A marca Sartorius Werke é notória na fabricação desse tipo de instrumento e sua história começou na década de 1870, quando o engenheiro alemão Florenz Sartorius (1846-1950) desenvolveu, a partir de peças de alumínio, uma balança leve, de braços curtos, fixada em sua própria base dentro de uma caixa de vidro e madeira. Tal modelo contrariava a linha de produção vigente, adotada pelos demais fabricantes, com modelos leves, de braços mais longos e removíveis da caixa (AFONSO; SILVA, 2004, p. 1024).

Conforme Maria Augusta André (2012, p. 151) explica em seu trabalho, instrumentos compreendidos como patrimônio e incorporados às coleções museológicas recebem tal significado devido às suas propriedades técnicas e/ou científicas. Nesse sentido, após as etapas de levantamento bibliográfico, acomodação temporária, registro dos objetos apurados e pesquisa museológica, tanto na internet como no arquivo do IGeo, almeja-se dar continuidade ao trabalho e aprofundar a busca por informações junto aos docentes e demais pesquisadores, a fim de sensibilizar a comunidade acadêmica sobre a importância desses objetos e musealizar essa coleção.

\section{CONSIDERAÇÕES FINAIS}

Todas as informações reunidas nesse texto são fruto de pesquisa e do esforço em reunir esses objetos em um único lugar para protegê-los do descarte. A reunião de informações tem por objetivo verificar a relevância desses objetos como patrimônio a fim de garantir apoio institucional para sua preservação por meio da formação de uma coleção bem conservada, acondicionada e documentada.

A preservação e a pesquisa sobre o patrimônio de ciência e tecnologia representam um grande desafio que as instituições enfrentam no Brasil e no mundo. Diversas áreas das ciências sociais e humanidades podem se beneficiar com essa preservação, tendo acesso a documentos e insumos para a pesquisa acadêmica os quais podem auxiliar também instituições acadêmicas e de memória: institutos de pesquisa, universidades, museus, arquivos, entre outros. Assim sendo, o trabalho de preservação desse patrimônio traz visibilidade, referências e valor. Quanto à sociedade, ganha em cidadania, educação, memória e informação ao obter acesso a esses bens.

As informações reunidas até o momento visam dar respaldo a esses anseios ao, sistematicamente, selecionar, musealizar e preservar os objetos 
de ciência e tecnologia pertencentes ao IGeo. Com o decorrer da pesquisa, outras articulações e parcerias poderão ser feitas junto às administrações e aos professores, beneficiando a universidade como um todo.

É necessário aprofundar ainda mais a pesquisa com estudos futuros, mas almeja-se que esse trabalho tenha contribuído não só para a preservação da história da ciência produzida pela universidade, mas também para a memória institucional de seus cursos e departamentos que remontam à antiga Faculdade Nacional de Filosofia, no caso do curso de Geografia, e à Companhia de Formação de Geólogos, caso do curso de Geologia. A formação de uma coleção, dentro dos critérios museológicos, será um diferencial em relação às demais coleções que estão sob a guarda do Museu da Geodiversidade, sendo imprescindível estar vinculada a ele.

Ainda não é possível garantir que os objetos localizados até o momento ingressem definitivamente na coleção do Museu da Geodiversidade. Houve uma intervenção no processo de descarte para possibilitar a análise e pesquisa desses objetos e, a partir das informações levantadas, atribuir valor patrimonial àqueles que forem relevantes para a pesquisa geocientífica desenvolvida na Universidade Federal do Rio de Janeiro.

Com os desdobramentos da pesquisa, a divulgação e as conversas com professores e pesquisadores surgiram novas possibilidades de doação de objetos que não estão mais em uso nos laboratórios com potencial para integrarem o acervo que está sendo constituído. Interferir em um processo que possivelmente resultaria no descarte desses objetos é um dos méritos desta pesquisa. Tais doações impulsionam o desenvolvimento de uma política de aquisição de acervos que, junto à demanda por um local adequado de acomodação, representarão os futuros desafios a serem superados pela instituição.

\section{REFERÊNCIAS}

AFONSO, Julio Carlos; SILVA, Raquel Medeiros da. Evolução da balança analítica. Quim. Nova, v. 27, n.6, p. 1021-1027, 2004. Disponível em: https://www.scielo.br/pdf/qn/v27n6/22296. pdf. Acesso em: jun. de 2020 .

ANDRÉ, M. A. Conhecer para preservar: práticas de socialização de acervos em museus de ciência e tecnologia. In: SILVA, M. C. S. M.; BARBOZA, C. H. M. (Org.). Acervos de ciência e tecnologia no Brasil: preservação, história e divulgação.v.1.Rio de Janeiro: MAST, 2012. p. 131-18o.

CASTRO, Aline Rocha de Souza Ferreira de; GRANATO, Marcus. Inventário de objetos relacionados ao patrimônio cultural de ciência e tecnologia no Instituto de Geociências (IGEO/ UFRJ): Resultados Parciais. In: XIX ENCONTRO NACIONAL DE PESQUISA EM CIÊNCIA DA INFORMAÇÃO - ENANCIB 2018 GT- 9 - Museu, Patrimônio e Informação. Londrina, 2018. 
DESVALLÉES, André; MAIRESSE, François (Ed.). Conceitos-chave de museologia. São Paulo: Icom: Armand Colin, 2013.

GRANATO, Marcus; LOURENÇO, Marta C. Reflexões sobre o patrimônio cultural da ciência e tecnologia na atualidade. Revista Memória em Rede, v. 4, p. 90, 2011.

GRANATO, Marcus; RIBEIRO, Emanuela Sousa; CERAVOLO, Suely, HANDFAS, Ethel Rosemberg. Cartilha de orientações gerais para preservação do patrimônio cultural de C\&T: Museu de Astronomia e Ciências Afins (MAST). Rio de Janeiro: MAST, 2013.

GRANATO, Marcus; SANTOS, Leandro Rosa dos; MIRANDA, Luiz Roberto M. de. Estudos sobre a conservação de instrumentos científicos históricos no Museu de Astronomia e Ciências Afins - MAST. Cadernos do CEOM, Chapecó, v. 18, n. 21, p. 243-274, 2005.

JULIÃO, Letícia. Pesquisa histórica no museu. In: CADERNO de diretrizes museológicas I. Brasília, DF: MinC/Iphan/Departamento de Museus e Centros Culturais. Belo Horizonte: Secretaria de Estado de Cultura de Minas Gerais, Superintendência de Museus, 2006. p.93-105.

SCIENCE museum collection: Richard Frères. [s.d.] Disponível em: https://collection. sciencemuseumgroup.org.uk/people/cp1659o/richard-freres. Acesso em: jun. 2020.

SILVA, Maria Celina Soares de Mello; BARBOZA, Christina Helena da Motta. Introdução: reflexões sobre os acervos de C\&T no Brasil. In: Acervos de ciência e tecnologia no Brasil: preservação, história e divulgação. Rio de Janeiro: MAST/MCTI, 2012. p.15.

SILVA, Shirley Maciel; DA SILVA, Gislene Rodrigues; NUNES, Daniele Vieira. Formação e gestão de coleção de instrumentos científicos do Departamento de Física. Instituto de Ciências Exatas da Universidade Federal de Minas Gerais/UFMG. In: ANAIS do SNBU, [S.1.], 2016. p.5.

TAUB, Liba. On scientific instruments. Studies in History and Philosophy of Science, v.40, p.337-343, 2009.

UNIVERSIDADE FEDERAL DO RIO DE JANEIRO. Departamento de Meteorologia. Apresentação. [s.d.]. Disponível em: https://meteorologia.igeo.ufrj.br/dpto/index.htm . Acesso em: jun. 2020.

\section{(cc) $\overline{\mathrm{E} Y \mathrm{YHo}-\mathrm{Ba}}$}

